\title{
Towards Understanding the Effect of Voice on Human-Agent Negotiation
}

\author{
Joanna Mania ${ }^{1,2}$, Fieke Miedema ${ }^{1}$, Rose Browne ${ }^{2}$, Joost Broekens ${ }^{2}$ and Catharine Oertel ${ }^{1}$ \\ ${ }^{1}$ Interactive Intelligence, Delft University of Technology, \{j.mania,f.e.g.miedema,c.r.m.m.oertel\}@tudelft.nl \\ ${ }^{2}$ Afective Computing, Leiden University, \{d.j.broekens, r.b.browne\}@liacs.leidenuniv.nl
}

\begin{abstract}
Virtual agents are increasingly being used for communication training such as public speaking-, job interviews-, as well as negotiation training. In these use-cases the agent is generally taking on the role of interviewer and its behaviour is altered according to the nonverbal cues of its human interlocutor. However, understanding how the agent's non-verbal cues influence human behaviour, perception or interactions outcomes is equally important. This contributes to appropriate behaviour generation in agents, but also to our understanding of the intricate interplay of non-verbal behaviours on human perception and interaction outcomes.

This study focuses specifically on the perception of vocal dominance in human-agent negotiation. Earlier research showed that the perception of dominance influences decision making in the course of negotiations, as do concessions tactics. However, the effect of voice as well as the effect of the negotiator type in this regard have been so far under-explored. To close this gap, an online experiment was conducted, in which a total number of 121 participants negotiated with conversational agents displaying either low or high vocal dominance, and an individualistic or neutral concession tactic. The results showed that when taking into account the self-reported type of negotiator, significant differences caused by vocal dominance were evident in regard to the number of negotiation rounds and perceived fairness. The number of rounds was significantly higher for the competitive participant type negotiating with the low vocal dominance agent, and the perceived fairness was significantly lower with the collaborative participant type negotiating with the high vocal dominance agent.
\end{abstract}

\section{KEYWORDS}

Negotiation; Virtual Agents; Vocal Dominance; Concession Tactic

\section{INTRODUCTION}

With virtual agents and social robots becoming more and more embedded into our daily lives, it is important to understand the effects of agents' non-verbal behaviours on interaction outcomes. One area in which the use of virtual agents is increasingly being explored, is the area of negotiation training [19]. This paper explores human-agent negotiation, seen as a dynamic and complex interaction between a human and an autonomous agent in an attempt to reach an agreement. By establishing favorable negotiation outcomes, with time-saving benefits and limited negative effects, autonomous negotiation agents have proven to reduce negotiation

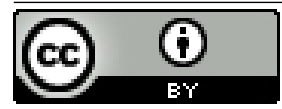

This work is licensed under a Creative Commons Attribution International 4.0 License. IVA '20, October 20-22, 2020, Virtual Event, Scotland Uk

(C) 2020 Copyright held by the owner/author(s).

ACM ISBN 978-1-4503-7586-3/20/09.

https://doi.org/10.1145/3383652.3423896 efforts, facilitate negotiation training and provide an advantage to less skilled negotiators [9].

A negotiation comprises several elements ranging from negotiation tactic to personality. Traditionally, research involving humanagent negotiation has focused on the effect of differing negotiation tactics. A negotiation tactic is formally defined as a concessionmaking model that is a function of time passing between the start of the negotiation and the endpoint, used to decide on the offer value given at a specific moment of the negotiation [17]. Ranging from no concession on one extreme to complete concession on the other one, these different tactics have shown to result in differences in negotiation perception and outcomes [40].

In recent years an increasing amount of research has additionally focused on endowing virtual negotiation agents with social skills comparable to humans [20]. One such example is the skill to adapt the tactic of the agent to the behavior displayed by the human negotiator [31]. In this line of research considerable attention has also been given to the agent's ability to express social signals, such as dominance. By implementing multi-modal non-verbal cues, such as body posture [35], facial expressions [13], eye gaze, head tilt [6] and turn-taking [15], it was shown that dominance, as a signal of power, can affect both the course and outcome of negotiations [7, $35,46]$.

While studies focusing on the implementation of non-verbal behaviours repeatedly stress the importance of the emotional quality of the voice, this signal has seldom been studied explicitly. Additionally, the negotiator's perception of vocal cues has to our knowledge, not been investigated with regards to his/her attitude towards the negotiation process itself. Taking this factor into account is however important as the personal attitude towards the negotiation process can differ per person, ranging from extreme negotiation avoidance to high engagement, which might result in different responses to a dominant opponent $[22,46]$. To our knowledge, no previous studies investigated the perception of vocal dominance on human-agent negotiation as a separate variable.

The main aim of this study is therefore to explore the effects of a conversational agent's vocal dominance and negotiation tactics on negotiation outcomes and perception. We are furthermore taking into account the human's self-reported negotiator type. The research question is set up as follows: What are the effects of vocal expression of dominance and concession tactics on the outcomes and perception of the human-agent negotiation, and are these effects influenced by the self-reported negotiator type?

This paper is organised in the following manner. First, the theoretical background and hypotheses are presented, followed by a methodology section. Next, we present the results and their discussion in the light of the current state-of-art. We conclude with limitations and suggestions for further research. 


\section{BACKGROUND}

\subsection{Vocal Expression of Dominance in Humans}

Dominance can be defined as a rational and expressive communication behavior to influence and extend one's power [16]. Vocal expression is considered a fundamental element of the communication process and functions as an information source for the conversational partner. In a study on the acoustic perception of emotional vocalizations, Banse and Scherer [5] have demonstrated that listeners were able to infer attitudes and affective states of the speaker based on acoustic features only and do it with an accuracy greater than expected by chance. Vocal variability, loudness, interruptions, pausing, rate of speech, pitch and vocal relaxation were shown to be essential aspects of conveying vocal dominance [21]. However, some of the vocal qualities mark it more effectively than the other ones. Tusing and Dillard [39] as well as Wang et al. [42] found that fundamental frequency serves as a predictor for the perception of dominance of the male voice, but not of the female voice. Investigation on the perceived degree of male dominance by Puts et al. [34] showed that voice recordings with a lowered fundamental frequency $\left(F_{0}\right)$ or formant frequencies $\left(D_{f}\right)$, were associated with a person who was more dominant socially and physically. Not surprisingly, the opposite was found in recordings where those parameters were raised [8]. The influence of voice was also investigated in relation to the political leadership capacity by Klofstad [26] Responses to the digitally manipulated voice recordings created to encourage participants to take part in elections, indicated that person with a lower-pitched voice, either female or male, was more likely to obtain a position of a leadership. Charfuelan et al. [11], by studying the perception of dominance through voice quality and prosody in group settings, showed that the group member who was perceived as the most dominant, had the tendency to speak with a higher voice volume. In contrast the person who was seen by others as the least dominant, used a lower voice volume while speaking. Contrasting these findings, Page and Balloun [32] found that the higher the voice volume, the higher the perception of aggressiveness. Surprisingly, modification of voice volumes did not cause any meaningful differences in perception of dominance or self-confidence. Instead, the low voice volume was rated as mature and sincere, as well as it increased the desires to collaborate with the speaker in a working settings [32].

2.1.1 Effect of Dominance on Negotiation Outcomes. Regarding human-human interactions, a friendly and warm communication style with a high degree of politeness signals low resource power and is perceived as low in dominance [3]. The norm of reciprocity predicts that such behavior will be perceived as open-handed and foresees that the opponent would reward it with warmth, generating positive negotiation outcomes [18]. Furthermore, behavior characterised by a sense of agreeableness, facilitating an open attitude towards the opponent, increases the likelihood of achieving agreements [44]. At the same time however, it also generates a risk of being perceived as less competent and easier to exploit. This can lead to the negotiation process taking more time, as the opponent might see more chances to achieve their own interests and behave egocentrically [38]. In human-human e-negotiations, Belkin et al. [7] showed that the linguistic expression of happiness was associated with submissiveness whereas the linguistic expression of anger with dominance. A behavior being perceived as more dominant achieved higher individual negotiation gains [7] and behavior taking on a tough and firm communication style resulted in higher economic outcomes and more beneficial counteroffers [25]. In line with this are findings in a multi-modal setting, where in human-agent negotiations virtual agents expressing dominant hand gestures and body postures convinced elderly more often to follow their suggestions than submissive agents [35]. However, none of these references investigated the perception of vocal dominance on negotiation in the context of human-agent interaction. Based on the presented findings, we propose the following hypothesis from the assumption that the effect of multi-modal expressions of dominance by humans on negotiation outcome will be similar to the vocal expression of dominance by agents: H1. The negotiations with a "low vocal dominance" agent will result in a (a) higher number of agreements, (b) higher number of rounds, (c) a lower utility of the agreement and $(d)$ lower best utility offered than the "high vocal dominance" negotiations.

2.1.2 Effect of Dominance on Negotiation Perception. In humanhuman negotiation, Welsh [45] presented a number of characteristic to judge whether the decision-making process or dispute solution is fair. The process was highly likely to be evaluated as fair if a person was given a room to speak, felt treated with consideration (i.e. being listened and understood), in a fair manner and with respect. This kind of approach to negotiation, by facilitating an equal distribution of resources and meeting interest of both parties, increases an overall feeling of satisfaction with the negotiation process [36]. The effect of fairness on negotiator satisfaction was confirmed in the context of Negotiation Support Systems (NSS)facilitated negotiation. It revealed that perceived fairness, next to the the degree of perceived collaborative atmosphere and perceived control, was a better predictor of negotiator satisfaction than negotiation outcomes [43]. Nevertheless, the degree of satisfaction with the outcome enhances the motivation to sustain the relationship and therefore has a positive effect on the willingness to renegotiate [37]. In the human-agent negotiation study of Prajod et al. [33], where emotions were implemented through non-verbal behavior, negotiation satisfaction was rated higher when interacting with a warm than a cold agent, as well as the willingness to renegotiate with him. Taking the assumption that perceived warmness and low dominance are highly correlated concepts, which we will verify through a pre-test in the following experiments, we are putting forward the following hypotheses: H2. The negotiations with a "low vocal dominance" agent will lead to a (a) higher degree of satisfaction, (b) higher willingness to renegotiate and (c) higher perceived fairness of the negotiation than the "high vocal dominance" negotiations.

\subsection{Concession Tactics in Negotiation}

The agents' negotiation tactics employed in this study belong to the time-dependent group of tactics, and are defined as individualistic and neutral [40]. The first one resembles competitive negotiation behavior and is characterised by the delayed concession that drops largely when the negotiation is close to the deadline, at the risk of reaching no agreement. The latter one makes small concessions at a constant rate throughout the whole negotiation process, resembling collaborative behavior (Figure 1). In both cases, the concessions are 


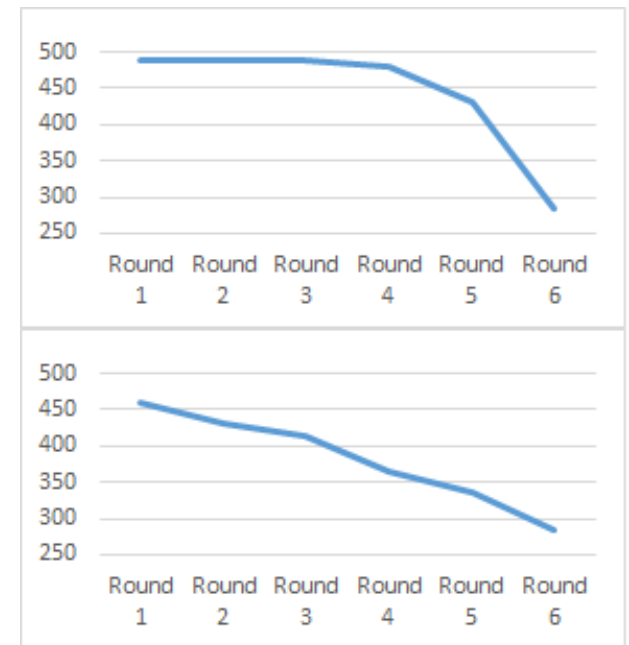

Figure 1: Concession Tactics: Individualistic (above) and Neutral (below)

determined in terms of the utility of the agent's offers and the time passed, without accounting for opponent modeling.

2.2.1 Effect of Concession Tactics on Negotiation Outcomes. An experimental study of Vahidov [40] on software negotiation tactics showed that large first concessions generated expectations of further significant concessions on the part of the agent and small concession by the opponent. Neutral agents - conceding gradually over time - created a sense of openness that invited to invest more time into the negotiation process, reached proportionally more agreements than agents using the individualistic tactic. At the same time, it has been shown that the individualistic tactic received more favorable offers from the opponent and outperformed the neutral tactic in terms of utilities as the negotiation agreements resulted in agreements with a higher utility than the neutral tactic $[2,40]$. Therefore, it is expected to find confirmation of the following hypothesis: H3. The individualistic tactic will lead to (a) a lower number of agreements, (b) a lower number of rounds, (c) a higher utility of the agreement and (d) a higher best utility offered than the neutral tactic.

2.2.2 Effect of Concession Tactics on Negotiation Perception. In the context of human-human negotiations, no meaningful differences have been found in the effects of concession timing on negotiation perception. Here both, gradual concession and delayed concession brought equivalently good results in comparison to early concession strategy [27, 37]. Nevertheless, when looking at the satisfaction in particular, the time invested in acquiring a product, impacted positively the level satisfaction with a product $[10,27]$. This finds confirmation in the human-agent negotiation context. When an agreement was reached, an individualistic tactic resulted in a higher degree of satisfaction when compared to a neutral or yielding tactic [47]. In regards to the perceived fairness in general, it has been shown that an adjustment of price in the course of the negotiation process had positive effect on the perception of fairness [28], however no previous studies has assessed this in the context of human-agents negotiations. In light of above-mentioned, the following hypotheses have been derived: H4. The individualistic tactic will lead to (a) a greater satisfaction, (b) lower perceived fairness and (c) a higher willingness to renegotiate than the neutral tactic.

\subsection{Negotiator Type as a Covariant}

Various degrees of competitiveness and need for control lead to different responses in the context of human-human negotiations. When considering the negotiator type in the human-human negotiations, it has been proven that more value was created during negotiations between opposite types of negotiators, than in the negotiations where both parties acted submissively or dominantly. A pair of dominant negotiators - striving for control - struggled to reach an agreement, whereas a pair of submissive negotiators was unable to set direction in the negotiation and little was accomplished[24]. In contrast, by taking contrasting approaches it was possible to improve coordination, satisfy priorities and generate positive feelings [46]. Depending on the negotiation framing, it has been shown that the collaborative type performed better and achieved beneficial joint outcomes, if the task was to minimize expenses. The competitive type proved to gain higher outcomes when the task was maximize profit [12]. The effect of opponent's negotiation type has been also researched in the context of human-agent negotiations. Automatic adaptation of agent's concession tactic and the offer acceptance to the human negotiator type allowed to obtain higher utility values agreements, improving the efficiency of negotiated agreement and prevent from exploitation by the opponent, when compared to both human player and other automated agents, making the process of negotiation more productive [23, 31]. Based on these findings, it is expected that the self-reported negotiator type (competitive or collaborative) will affect the perception of the agent's vocal dominance, and consequently impact the negotiation outcomes and perception.

\section{CONTRIBUTIONS}

By assessment of the above-mentioned effects, this research contributes to understanding the expressive power of voice as an autonomous source of dominance. To our knowledge no previous study has looked i) at human-agent negotiation focusing on the vocal dominance dimension ii) at the possible interaction between vocal dominance and concession tactics and iii) the moderating effect of the negotiator type on both vocal dominance and concession tactics. Our study extends the body of literature by showing that the perception of dominance, when expressed by an agent through voice, is influenced by the self-perceived negotiator type.

\section{METHOD}

\subsection{Experimental design}

To examine the influence of vocal expression of dominance, concession tactics and the moderating effect of the self-reported negotiation type on the negotiation outcomes and perception, an online experiment was set up. A $2 \times 2$ factorial design creating four conditions was implemented, using the two independent variables vocal dominance (high vocal dominance vs low vocal dominance) and concession tactic (individualistic vs neutral) as within-subject factors. The self-reported negotiator type (competitive vs collaborative) is an unmanipulated between-subject factor.

4.1.1 Main experiment. The online experiment was implemented in Qualtrics and structured in three parts. Firstly, written consent and an explanation of the study aim were presented, followed by a set of demographic questions. Secondly, each participant was 
asked to play a negotiation game, where he or she was randomly exposed to one of the four experimental conditions. In the last part, a question about the self-perceived negotiator type was presented. The negotiation game was an adapted version of one-on-one "fixed pie" negotiation system $[33,41]$. The participant played the role of a customer in a mobile phone store, whose task was to negotiate with a virtual agent, playing the role of a salesman. Objects of negotiations were: price, warranty and service agreements. All three issues were assigned points on seven levels. The total amount of points was calculated as the sum of the products of the level number and the assigned points per level for the corresponding issue:

Utility participant $=$

$50 \times$ PriceLevel $+15 \times$ WarrantyLevel $+30 \times$ ServiceLevel

\begin{tabular}{|c|c|c|}
\hline Price & Warranty & Service \\
\hline$€ 250$ (0 points) & 1 month ( 0 points $)$ & 1 month (0 points) \\
\hline$€ 245$ (50 points) & 2 months (15 points) & 2 months (30 points) \\
\hline$€ 240$ (100 points) & 3 months (30 points) & 3 months (60 points) \\
\hline$€ 235$ (150 points) & 4 months (45 points) & 4 months (90 points) \\
\hline$€ 230$ (200 points) & 5 months (60 points) & 5 months (120 points) \\
\hline$€ 225$ (250 points) & 6 months (75 points) & 6 months (150 points) \\
\hline$€ 220$ (300 points) & 7 months (90 points) & 7 months (180 points) \\
\hline
\end{tabular}

Each offer of the agent was communicated through an audio clip. The participant was given three response options: "I want to accept the offer" "I want to end the negotiation without an agreement" or "I want to propose a counteroffer" (Figure 2). The goal of the game was to reach an agreement while scoring as many points as possible. To engage participants in the negotiation process, a bonus of 50 euro was granted to the participant reaching the agreement with the highest point value. To prevent overly-competitive behavior, the agent was set to withdraw from the negotiation process, if no agreement was reached after the end of the sixth round. If the participant acted overly-collaborative, by conceding too much, a high-scoring agreement was out of reach.

\subsection{Stimulus material}

4.2.1 Voice generation. To alternate the vocal dominance of the agent across the two negotiation tactics, four sets of voice recordings were created: 1) high vocal dominance $\mathrm{x}$ individualistic tactic, 2) high vocal dominance $x$ neutral tactic, 3) low vocal dominance $x$ individualistic tactic and 4) low vocal dominance $\mathrm{x}$ neutral tactic. A native British English voice actor was recruited due to geographical closeness and the fact that this language is taught as a second language throughout Europe. To achieve distinguishable vocal traits, the voice actor adhered to a set of instructions drawn upon literature on voice perception [21]. To convey high vocal dominance, the actor was requested to produce the following voice qualities: low pitch, increased amplitude and staccato, complemented by acted personality traits such as self-oriented, direct, energetic and slightly aggressive. For the low vocal dominance, it altered to voice qualities such as high pitch, regular to soothing amplitude, 'singing' voice and less energetic, complemented by acted personality traits like friendly, accessible and calm. Except for the different offer values assigned for each of the two negotiation tactics, all recordings followed the same script and were recorded in a professional studio, using a sound-proof room and microphone. The script was an adapted version of a conversational dialogue for an agent-seller scenario [33]. The post-processing of audio clips involved the removal of white noise and equalizing the loudness (measured in $\mathrm{Db}$ ) across all recordings with the Audacity tool.

Listen to the first offer:

$\longrightarrow 0$ 0:00/ 0:13

\begin{tabular}{|c|c|c|}
\hline Price & Warranty & Services \\
\hline$€ 250$ (0 points) & 1 month (0 points) & 1 month (0 points) \\
\hline$€ 245$ (50 points) & 2 months (15 points) & 2 months (30 points) \\
\hline$€ 240$ (100 points) & 3 months (30 points) & 3 months (60 points) \\
\hline$€ 235$ (150 points) & 4 months ( 45 points) & 4 months (90 points) \\
\hline$€ 230$ (200 points) & 5 months (60 points) & 5 months (120 points) \\
\hline$€ 225$ (250 points) & 6 months (75 points) & 6 months (150 points) \\
\hline$€ 220$ (300 points) & 7 months (90 points) & 7 months (180 points) \\
\hline
\end{tabular}

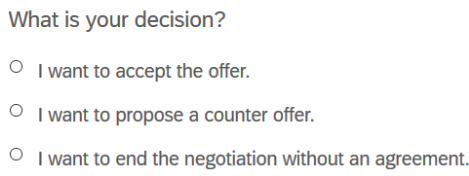

Figure 2: The negotiation interface in Qualtrics

4.2.2 Vocal Dominance Validation. To validate the stimulus material, a small-scale online voice perception study was set up. A group of 16 participants recruited from the direct personal network of the researchers was exposed to a sequence of four snippets of low vocal dominance recordings and a sequence of four snippets of high vocal dominance recordings, in random order. To obtain an objective assessment of the manipulated voice stimuli and prevent bias towards submissiveness and/or dominance, participants were asked to provide qualitative feedback first, i.e. describe shortly the character attributes associated with that person in free form text. Next, they were asked to rate dominance, competence and warmth on the three 9-point bipolar scales: submissive-dominant, incompetent-competent and cold-warm. Lastly, the subjects were asked to indicate which of the agents they preferred to negotiate with as well as which of the two agents was more likely to win in a physical fight and convince his peers in a group meeting. All responses were used as indicators of vocal dominance [34]. This group of participants was excluded from the main experiment. Outcomes of the pre-test are reported in the Results section.

4.2.3 Negotiation tactic. Both negotiation tactics were set up to have different utility values for each bid as to differentiate them clearly, but ended with the same utility value. The reservation value was also equal for the two tactics: each agent accepted the participant's offer when the utility of the bid of the participant was higher than 285 points, in favour of the agent. If the participant's offer was below that threshold, the agent proposed a counter-offer [33]. The individualistic tactic was set to start with a very low offer and keep oscillating around 80 points in the first four rounds. In the fifth round, it increased to 220 points to end with the last offer worth 285 points. The neutral negotiation tactic started with an offer worth 110 points for the participant, and was set to gradually grow in each round, worth respectively 140, 155, 205, 235 to end with the final offer of 285 points. 


\subsection{Measurements}

The measurements of negotiation outcomes were operationalized as follows: number of reached agreements showed the willingness to achieve an agreement expressed as the number of accepted offers by either the participant or the agent; utility of the agreement was the number of points calculated by the Utility Participant formula it equaled 0 if no agreement was reached; best utility offered to the agent was defined as the highest utility offered to the agent amongst all bids proposed by the participant; number of rounds indicated the pace of conceding and equaled the number of bids offered by the participant before the negotiation stopped. The evaluation of the negotiation itself was based on three sets of statements measured on a 7-point Likert scale: perceived fairness of the negotiation ("The seller negotiated fair and reasonable", "The seller aimed to get an unfair advantage"), outcome satisfaction ("I feel satisfied with the outcome of the negotiation", "I negotiated a good deal.") and willingness to renegotiate ("I am willing to negotiate with this seller again", "I would recommend negotiation with this seller to friends and family"). Lastly, the participants were asked to indicate whether they perceive themselves in general as the competitive or collaborative negotiator type.

\section{RESULTS}

Data was obtained using Qualtrics and pre-processed for analysis in Python. The pre-processing included data cleaning, transforming variable types and re-coding labels into binary values. The analysis was conducted in SPSS. The statistically significant results for $\alpha=$ 0.05 and $\alpha=0.01$ are indicated with * and ${ }^{* *}$ respectively.

\subsection{Vocal dominance - pretest}

The pretest aimed to verify whether the low and high vocal dominance in the audio clips was perceived as such. The participants were first asked to describe the characteristics of the two different voices in three words. Secondly, they were asked to rate both voices on three scales: level of dominance, level of warmth and level of competence. The conditions were presented to the participants in random order: either the high vocal dominance voice was presented first, or the low dominance voice was. To describe the high dominance voice, the participants used words related to high dominance ("Persuasive", "Aggressive", "Dominant"), high competence ("Accurate", "Rational", "Quick") and low warmth ("Cold", "Distant", "Unapproachable"). The words used to describe the low dominance voice were low dominance ("Insecure", "Timid", "Collaborative"), high competence ("Professional", "Correct", "Respectful") and high warmth ("Friendly", "Warm", "Enthusiastic"). Both outcomes align with how low and high dominance was identified in past studies [4]. Based on 16 data points, the high dominance voiced agent scored on average $6.8(s d=1.5)$ on the dominance scale, whereas the low dominance voiced agent was rated $3.9(s d=1.3)$. In turn, the low dominance voiced agent was rated $5.8(s d=2.4)$ on warmth, while the high dominance voiced agent scored only $3.1(s d=1.7)$. The ratings of the agents showed that they were both perceived as equally competent ( 5.5 and $5.6, s d=2.5$ and $s d=2.5$ ). The outcomes are portrayed in Figure 3. Furthermore, 81.3\% thought the high dominance voiced agent was more likely to win in a physical fight and $62.5 \%$ found this agent better at convincing his peers in a meeting, which is consistent with findings on acoustic qualities of

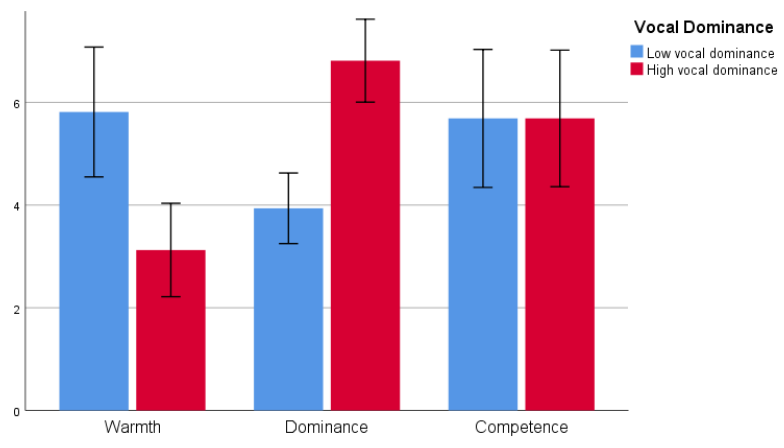

Figure 3: Bar chart of the Warmth, Competence and Dominance scores

dominance [34]. Therefore, the pretest analysis confirmed that the low and high vocal dominance of agents was perceived as such, and allowed to carry out the remaining part of the study using these voice recordings.

\subsection{Negotiation experiment}

A total number of 194 participants took part in the online experiment. For the data analysis, only the 121 subjects who completed the negotiation task and answered the post questions were selected.

5.2.1 Sample. A total number of 121 participants (57\% female) predominantly in the age group of 25-34 years (55.4\%), were representatives of the following language groups: Dutch $(n=73)$, Polish $(n=9)$, Italian $(n=6)$, German $(n=5)$, English $(n=5)$, Spanish $(n=4)$, Chinese $(n=4)$ and other $(n=15)$. The majority has completed a Bachelor's degree (50.4\%) and reported to be a student (48.8\%). $82.7 \%$ admitted having a neutral or positive attitude towards negotiation. 5.2.2 Reliability analysis. The homogeneity and reliability of the scales were verified: perceived fairness had a Cronbach's $\alpha=0.528$, the outcome satisfaction a Cronbach's $\alpha$ of 0.835 and the willingness to renegotiate a Cronbach's $\alpha$ of 0.734 . The scores of the outcome satisfaction and willingness to renegotiate scales were calculated using the mean of the two items. The two items of perceived fairness were tested separately throughout the rest of the analysis, instead of combining them in one scales, as a score below 0.7 is considered unreliable.

5.2.3 Sample variability. To validate the even distribution of demographic variables amongst the four conditions, a Chi-Square test was used to assess the associations between age, gender and highest degree and the four conditions. To ensure that no tests were carried out between groups smaller than 10 people, the following variables were recoded: Age into '18-24 years old' ( $n=32)$, '25-34 years old' ( $n=67)$ and ' $35+$ ' $(n=22)$ and Highest degree into 'Below bachelor's degree' $(n=24)$, 'Bachelor's degree' $(\mathrm{n}=61)$ and 'Master's degree or doctorate' $(n=36)$. The gender of the participants did not differ per category, $\chi^{2}(3, \mathrm{~N}=121)=3.17, \mathrm{p}=.37$, neither for the age of participants, $\chi^{2}(3, \mathrm{~N}=121)=1.04, \mathrm{p}=.79$, or the level of education of the participants, $\chi^{2}(6, \mathrm{~N}=117)=3.62, \mathrm{p}=.73$.

5.2.4 Analysis of self-reported negotiator type and negotiation behavior. The self-reported negotiator type was an unmanipulated between-subject factor, which was analysed on coherence with the displayed actions of the participants. There were 68 participants 
Table 1: Results of T-Test on negotiation behavior

\begin{tabular}{lrr}
\hline Variable & Test statistic & p-value \\
\hline \# Agreements & -5.158 & $0.000^{* *}$ \\
\# Rounds & 4.723 & $0.000^{* *}$ \\
Utility first bid & 4.228 & $0.000^{* *}$ \\
Average utility & 5.983 & $0.000^{* *}$ \\
Utility difference & 2.822 & $0.005^{* *}$
\end{tabular}

who identified themselves as collaborative and 53 as competitive. In the context of the negotiation experiment, we analysed the competitive and collaborative behavior of participants on five variables: the amount of agreements, the amount of negotiation rounds, the utility of the opening bid, the average utility of all the proposed bids and the average utility difference between bids. On average, the participants viewing themselves as competitive reached an agreement $42.59 \%$ of the time $(s d=0.07)$ and negotiated for 4.11 rounds $(s d=0.23)$. The collaborative participants reached an agreement much more often, namely in $84.29 \%$ of the time $(s d=0.04)$. Their negotiations also lasted fewer rounds: 2.70 rounds $(s d=0.20)$. Both results are in line with research that showed that the amount of egocentrism of the negotiator predicts the length of time it would take to reach an agreement, as well as reducing the ability to come to an agreement [38]. When comparing the utilities of the first bid, the participants who considered themselves collaborative started with a bid that was on average 91.70 points lower than those who considered themselves competitive (344.62 points, $s d=130.54 \mathrm{vs}$ 436.32 points, $s d=111.06)$. Their average utility of all the bids in the negotiation experiment was also different: $322.92(s d=79.43)$ for the collaborative participants and $407.28(s d=75.72)$ for the competitive participants. Finally, the collaborative participants lowered their offer an average -60.82 points $(s d=79.83)$ between bids, meaning they conceded on average $\approx 61$ points each negotiation round. This number was on average much lower for the competitive participants, who only conceded $\approx 33$ points $(s d=80.07)$.

Once validated that the self-reported negotiator type matched the actual negotiation behavior, we tested for differences between the groups with independent samples T-test. As presented in Table 1, the groups were also statistically significantly different in their behavior on the five variables. Equal variances were assumed, as Levene's test showed significance levels above 0.05 .

5.2.5 Analysis of main affects and effect of self-reported negotiator type. To test the hypotheses on the effects of vocal dominance, concession tactic and self-reported negotiator type, a two-way MANCOVA was performed with vocal dominance and concession tactic as main variables and self-reported negotiator type as covariate.

The MANCOVA analysis showed that neither vocal dominance (Wilk's $\lambda$ multivariate $F(7,110)=1.181, \mathrm{p}=0.320$ ) nor agent negotiation strategy (Wilk's $\lambda$ multivariate $F(7,110)=1.009, \mathrm{p}=0.429$ ) had a significant overall effect. Interaction effects were not significant either. The only effect that was significant on individual outcome measures was the effect of negotiation tactic on perceived fairness $(F(1,116)=5.667, \mathrm{p}=0.019)$. This showed that an individualistic concession strategy was rated as less fair ( $m e a n=3.65, s d=0.19)$ than a neutral one ( $m e a n=4.25, s d=0.18$ ). A Mann-Whitney U test confirmed these findings showing only a significant effect of concession tactic on perceived fairness $(U=1412.5, \mathrm{p}=0.027)$. This confirmed the hypothesis that an individualistic tactic would lead to a lower perceived fairness $(\mathrm{H} 4 \mathrm{~b})$.
Table 2: Results Mann-Whitney U tests on negotiator type

\begin{tabular}{lll}
\hline Negotiation outcome & Test statistic & p-value \\
\hline Number of rounds & 996.50 & $0.000^{* *}$ \\
Utility agreement & 925.50 & $0.000^{* *}$ \\
Best utility offered & 1157.50 & $0.001^{* *}$ \\
\hline Negotiation perception & Test statistic & p-value \\
\hline Satisfaction outcome & 1402.50 & $0.035^{*}$ \\
Perceived fairness & 1363.50 & $0.019^{*}$ \\
Unfair deal & 1583.50 & 0.241 \\
Willingness to renegotiate & 1444.00 & 0.060
\end{tabular}

However, self-reported negotiator type significantly correlated with outcome measures in general (Wilk's $\lambda$ multivariate $F(7,110)=$ 6.012, $\mathrm{p}<0.001)$. To detail these results, we performed a specific non parametric tests (Mann-Whitney) to ensure the assumptions for the post hoc tests were not violated. These showed that a self-reported competitive negotiator is significantly related to a higher number of rounds ( mean $=4.11, s d=0.23$ versus mean $=2.70, s d=0.20$ ), a lower utility of agreement $($ mean $=95.00, s d=14.06$ vs mean $=$ 178.42 , $s d=12.36$ ), a higher best utility offered ( mean $=453.07, s d=$ 19.08 vs mean $=330.72, s d=16.78)$, a lower perceived fairness $($ mean $=3.59, s d=0.19$ vs mean $=4.21, s d=0.17)$, and a lower satisfaction outcome ( mean $=3.42$, $s d=0.22$ vs mean $=4.00, s d=0.20$ ). See Table 2 for an overview of the test statistics.

5.2.6 Potential interaction effects between perceived negotiator type and main variables. As there was such a large effect of self-reported negotiator type, we investigated potential interactions effects with vocal dominance and strategy. Again, we opted for non-parametric tests in order to not violate any assumptions in the post testing. We splitted the data file into two subsets (collaborative versus competitive) before performing the non-parametric tests for the effect of vocal dominance and concession tactic on the outcome variables. As the results in Table 3 show, there were indeed significant differences in both negotiation perception and negotiation outcome based on the vocal dominance expressed by the agent, when the type of negotiator (collaborative or competitive) is taken into account.

Vocal dominance influenced the number of rounds for the group that perceived themselves as competitive negotiators: negotiating with the high dominance agent caused the competitive group to end the negotiation with a lower amount of rounds than when negotiating with the low dominance agent. This was not the case for the collaborative group. The perceived fairness of the negotiation was significantly different for the group that perceived themselves as collaborative negotiators; negotiating with the low dominance agent resulted in a significantly higher perceived fairness than when negotiating with the high dominance agent. Self reported negotiator type did not seem to influence the effect of concession tactics.

\section{DISCUSSION}

The results of this study only to a small extent confirmed expectations derived from previous studies on human-human and humanagent negotiations. Following the findings of Belkin et al. [7], where dominance lead to higher gains, and the findings of Rosenthal [35], where dominant virtual agents were more persuasive, it was expected that the manipulated vocal dominance would lead to different negotiation outcomes. Our results did not confirm these (H1 
Table 3: Results of Mann-Whitney U tests for vocal dominance and concession tactic when split on negotiator type

\begin{tabular}{|c|c|c|c|c|c|c|c|c|}
\hline & \multicolumn{4}{|c|}{ Collaborative } & \multicolumn{4}{|c|}{ Competitive } \\
\hline & \multicolumn{2}{|c|}{ Vocal dominance } & \multicolumn{2}{|c|}{ Concession tactic } & \multicolumn{2}{|c|}{ Vocal dominance } & \multicolumn{2}{|c|}{ Concession tactic } \\
\hline & Test statistic & p-value & Test statistic & p-value & Test statistic & p-value & Test statistic & p-value \\
\hline Number of rounds & 573.00 & 0.955 & 519.00 & 0.458 & 228.50 & $0.027^{*}$ & 309.00 & 0.439 \\
\hline Utility agreement & 419.50 & 0.052 & 480.50 & 0.230 & 270.50 & 0.126 & 327.00 & 0.637 \\
\hline Best utility offered & 559.00 & 0.820 & 508.00 & 0.390 & 316.50 & 0.571 & 346.50 & 0.936 \\
\hline Satisfaction outcome & 505.00 & 0.368 & 533.50 & 0.580 & 318.00 & 0.588 & 275.50 & 0.174 \\
\hline Perceived fairness & 418.00 & $0.044^{*}$ & 478.00 & 0.207 & 340.00 & 0.884 & 246.50 & 0.059 \\
\hline Unfair deal & 529.50 & 0.543 & 567.50 & 0.894 & 312.00 & 0.510 & 321.00 & 0.584 \\
\hline Willingness to renegotiate & 535.00 & 0.599 & 515.00 & 0.435 & 321.00 & 0.628 & 322.50 & 0.610 \\
\hline
\end{tabular}

$a / b / c / d$ and $H 2 a / b / c$ rejected), which is not surprising due to expressiveness of implemented modalities. In this study, the level of dominance was inferred from the voice only - without additional cues as facial expression or gestures. As a consequence, the effects might have been milder than when the dominant behavior is implemented using multiple modalities. This would also be in line with Melo et al. [14], who found differences in participants' decision making in negotiation when varying emotional displays of virtual agents. Furthermore, while the individualistic tactic and neutral tactic differed on the average best utility offered by the agent to the participant, this did not influence any of the negotiation outcomes, such as utility of the agreement or the best utility offered by the participant (rejected $\mathrm{H} 3 \mathrm{a} / \mathrm{b} / \mathrm{c} / \mathrm{d}$ ). Additionally, our analysis found that participants perceived the individualistic tactic as less fair than the neutral tactic (accepted $H 4 b$ ). One might argue that, just as in human-human negotiations, the lack of flexibility with regards to value adjustment, might have led to a feeling that the opponent is set to achieve only his or her own goals. This, however, did not influence their satisfaction with the outcome, or their willingness to renegotiate (rejected $\mathrm{H} 4 \mathrm{a} / \mathrm{c}$ ).

As the scale of fairness had a low Cronbach's alpha, the perceived fairness variable related to the question "The seller negotiated fair and reasonable". The other question of the scale, "The seller aimed to get an unfair advantage" was perceived as not relating to the same concept. Reviewing the participants scores revealed that this might partly be related to the survey design. Unfair advantage was the only mirrored question, which caused some participants to score both a 7 for 'fair and reasonable' and 'unfair advantage'. Additionally, fairness in literature is often described as consisting out of three types of fairness: procedural fairness, process fairness and outcome fairness [1]. The question "the seller negotiated fair and reasonable" might have appealed to process fairness, while the "get an unfair advantage" could be related to "outcome fairness". Extending the scale to more questions and rephrasing the questions to scoring in the same direction could bring more insight into how the concept of fairness is perceived in this human-agent negotiation setting.

Last but not least, it is also worthwhile to note that the significant effect on an individual outcome measure was related to the perception of negotiation. This indicates that participants perceived a difference with regards to vocal dominance but that this differences affected participants in different ways based on their self-reported negotiator type. Specifically, when taking this into account, the analysis showed that the competitive type group ended the negotiation with significantly less rounds when negotiating with the high vocal dominance agent. A possible explanation is that competitive negotiators might have applied a hardball tactic also by ending the negotiation early, in the hopes that the agent would come back with a final and better offer. Alternatively, when interacting with the low vocal dominance agent, they felt there was more to gain, and hence negotiated more rounds with that agent. For the group that identified as collaborative negotiators, the perceived fairness was significantly lower when they negotiated with the high vocal dominance agent. This is interesting as the perceived fairness was not significantly different for the individualist tactic versus the neutral tactic for the collaborative group, while it was for the sample in general $(H 4 b)$. We conclude that the vocal dominance, more than the actual offers of the agent, influenced the fairness perception. Both results point in the direction of the influence of the negotiation style and/or personality of the participant on the perception of the vocal dominance and the effects on the negotiation.

\section{CONCLUSION AND FUTURE WORK}

The main goal of this study was to investigate if an agent exhibiting low or high dominance through vocal cues affects the negotiation outcome and whether there were any significant differences depending on self-perceived negotiator type. No main effects of vocal dominance or concession tactic were found, meaning that the negotiation outcomes and negotiation perception were not altered by our manipulation. We did find one effect on individual outcome measures, stating that the perceived fairness is affected by the concession tactic of the agent: the individualistic tactic is perceived as significantly less fair than the neutral tactic. Our findings also suggest that the modulation of vocal cues by themselves, without any additional visual information, is sufficient for conveying different degrees of dominance. It appears, however, that this difference in vocal dominance leads to a difference in negotiation action for participants that perceive themselves as competitive negotiators. Specifically, negotiating with the high dominance agent caused the competitive group to end the negotiation with a lower amount of rounds than when negotiating with the low dominance agent. The collaborative group did not behave differently during the negotiation with the high dominance agent, but perceived the agent as significantly less fair. In future studies it would be interesting to include additional multi-modal cues, such as gaze and facial expressions to our investigation of the perception of dominance in human-agent interaction. We are further aiming to explore multi-party settings, where gaze and prosody have already been shown to be related to the perception of engagement $[29,30]$. We are hypothesising that they might also proof useful for the modelling of dominance in such settings. In addition, endowing the conversational agent with further conversational capabilities could strengthen the agent's dominance effect and improve overall experience of immersiveness. In fact, in this study the audio clips had to be played manually, which might have negatively impacted the natural feel of the negotiation. Additionally, taking into account information regarding negotiation skills and experience, as well 
as preferred negotiation- and personality type could aid to better understand the participants' decisions and explain differences in negotiation outcomes and perception.

\section{REFERENCES}

[1] C. Albin. 1992. Fairness Issues in Negotiation: Structure, Process, Procedures and Outcome. IIASA Working Paper. IIASA, Laxenburg, Austria. http://pure.iiasa.ac. at/id/eprint/3612/

[2] Tim Baarslag, Katsuhide Fujita, Enrico H Gerding, Koen Hindriks, Takayuki Ito, Nicholas R Jennings, Catholijn Jonker, Sarit Kraus, Raz Lin, Valentin Robu, et al. 2013. Evaluating practical negotiating agents: Results and analysis of the 2011 international competition. Artificial Intelligence 198 (2013), 73-103.

[3] Samuel B Bacharach and Edward J Lawler. 1981. Bargaining: Power, Tactics and Outcomes. ERIC.

[4] Samuel B Bacharach and Edward J Lawler. 1981. Power and tactics in bargaining. ILR Review 34, 2 (1981), 219-233.

[5] Rainer Banse and Klaus R Scherer. 1996. Acoustic profiles in vocal emotion expression. Fournal of personality and social psychology 70, 3 (1996), 614.

[6] Nikolaus Bee, Stefan Franke, and Elisabeth Andreé. 2009. Relations between facial display, eye gaze and head tilt: Dominance perception variations of virtual agents. In 2009 3rd International Conference on Affective Computing and Intelligent Interaction and Workshops. IEEE, 1-7.

[7] Liuba Y Belkin, Terri R Kurtzberg, and Charles E Naquin. 2013. Signaling dominance in online negotiations: The role of affective tone. Negotiation and Conflict Management Research 6, 4 (2013), 285-304.

[8] Barbara Borkowska and Boguslaw Pawlowski. 2011. Female voice frequency in the context of dominance and attractiveness perception. Animal Behaviour 82, 1 (2011), 55-59.

[9] Joost Broekens, Maaike Harbers, Willem-Paul Brinkman, Catholijn M Jonker, Karel Van den Bosch, and John-Jules Meyer. 2012. Virtual reality negotiation training increases negotiation knowledge and skill. In International Conference on Intelligent Virtual Agents. Springer, 218-230.

[10] Richard N Cardozo. 1965. An experimental study of customer effort, expectation, and satisfaction. Journal of marketing research 2, 3 (1965), 244-249.

[11] Marcela Charfuelan, Marc Schröder, and Ingmar Steiner. 2010. Prosody and voice quality of vocal social signals: the case of dominance in scenario meetings. In Eleventh annual conference of the international speech communication association.

[12] Eva Chen and Gregory Kersten. 2010. Collaborators and competitors negotiating in profit and costs frames. InterNeg Res Pap 5 (2010), 10.

[13] Celso M de Melo, Peter Carnevale, and Jonathan Gratch. 2011. The effect of expression of anger and happiness in computer agents on negotiations with humans. In The 10th International Conference on Autonomous Agents and Multiagent Systems-Volume 3. International Foundation for Autonomous Agents and Multiagent Systems, 937-944.

[14] Celso M de Melo, Peter Carnevale, and Jonathan Gratch. 2012. The effect of virtual agents' emotion displays and appraisals on people's decision making in negotiation. In International Conference on Intelligent Virtual Agents. Springer, $53-66$.

[15] David DeVault, Johnathan Mell, and Jonathan Gratch. 2015. Toward natural turntaking in a virtual human negotiation agent. In 2015 AAAI Spring Symposium Series.

[16] Norah E Dunbar and Judee K Burgoon. 2005. Perceptions of power and interactional dominance in interpersonal relationships. Fournal of Social and Personal Relationships 22, 2 (2005), 207-233.

[17] Peyman Faratin, Carles Sierra, and Nick R. Jennings. 1998. Negotiation decision functions for autonomous agents. Robotics and Autonomous Systems 24, 3 (1998), 159 - 182. Multi-Agent Rationality.

[18] Alvin W Gouldner. 1960. The norm of reciprocity: A preliminary statement. American sociological review (1960), 161-178.

[19] Jonathan Gratch, David DeVault, and Gale Lucas. 2016. The benefits of virtual humans for teaching negotiation. In International Conference on Intelligent Virtual Agents. Springer, 283-294.

[20] Jonathan Gratch, David DeVault, Gale M Lucas, and Stacy Marsella. 2015. Negotiation as a challenge problem for virtual humans. In International Conference on Intelligent Virtual Agents. Springer, 201-215.

[21] Judith A. Hall, Erik J. Coats, Bala Cynwyd Pa, and Lavonia Smith Lebeau. 2005 Nonverbal behavior and the vertical dimension of social relations: A metaanalysis. Psychological Bulletin (2005), 898-924.

[22] James E Heyman, Yesim Orhun, and Dan Ariely. 2004. Auction fever: The effect of opponents and quasi-endowment on product valuations. Fournal of interactive Marketing 18, 4 (2004), 7-21.

[23] Koen Hindriks, Catholijn Jonker, and Dmytro Tykhonov. 2009. Using opponent models for efficient negotiation. In Proceedings of The 8th International Conference on Autonomous Agents and Multiagent Systems-Volume 2. 1243-1244.

[24] Leonard M Horowitz, Kelly R Wilson, Bulent Turan, Pavel Zolotsev, Michael J Constantino, and Lynne Henderson. 2006. How interpersonal motives clarify the meaning of interpersonal behavior: A revised circumplex model. Personality and Social Psychology Review 10, 1 (2006), 67-86.

[25] Martha Jeong, Julia Minson, Michael Yeomans, and Francesca Gino. 2019. Communicating with Warmth in Distributive Negotiations Is Surprisingly Counterproductive. Management Science 65, 12 (2019), 5813-5837.

[26] Casey A Klofstad, Rindy C Anderson, and Susan Peters. 2012. Sounds like a winner: voice pitch influences perception of leadership capacity in both men and women. Proceedings of the Royal Society B: Biological Sciences 279, 1738 (2012), 2698-2704.

[27] Seungwoo Kwon and Laurie R Weingart. 2004. Unilateral concessions from the other party: concession behavior, attributions, and negotiation judgments. Journal of Applied Psychology 89, 2 (2004), 263.

[28] Sarah Maxwell, Pete Nye, and Nicholas Maxwell. 1999. Less pain, same gain: The effects of priming fairness in price negotiations. Psychology \& marketing 16, 7 (1999), 545-562.

[29] Catharine Oertel, Kenneth A Funes Mora, Joakim Gustafson, and Jean-Marc Odobez. 2015. Deciphering the silent participant: On the use of audio-visual cues for the classification of listener categories in group discussions. In Proceedings of the 2015 ACM on International Conference on Multimodal Interaction. 107-114.

[30] Catharine Oertel and Giampiero Salvi. 2013. A gaze-based method for relating group involvement to individual engagement in multimodal multiparty dialogue. In Proceedings of the 15th ACM on International conference on multimodal interaction. 99-106.

[31] Yinon Oshrat, Raz Lin, and Sarit Kraus. 2009. Facing the challenge of human-agent negotiations via effective general opponent modeling. In Proceedings of The 8th International Conference on Autonomous Agents and Multiagent Systems-Volume 1. 377-384.

[32] Richard A Page and Joseph L Balloun. 1978. The effect of voice volume on the perception of personality. The journal of social psychology 105, 1 (1978), 65-72.

[33] Pooja Prajod, Mohammed Al Owayyed, Tim Rietveld, Jaap-Jan van der Steeg, and Joost Broekens. 2019. The Effect of Virtual Agent Warmth on Human-Agent Negotiation. In Proceedings of the 18th International Conference on Autonomous Agents and MultiAgent Systems. International Foundation for Autonomous Agents and Multiagent Systems, 71-76.

[34] David Andrew Puts, Carolyn R Hodges, Rodrigo A Cárdenas, and Steven JC Gaulin. 2007. Men's voices as dominance signals: vocal fundamental and formant frequencies influence dominance attributions among men. Evolution and Human Behavior 28, 5 (2007), 340-344

[35] Astrid M Rosenthal-von der Pütten, Carolin Straßmann, Ramin Yaghoubzadeh, Stefan Kopp, and Nicole C Krämer. 2019. Dominant and submissive nonverbal behavior of virtual agents and its effects on evaluation and negotiation outcome in different age groups. Computers in Human Behavior 90 (2019), 397-409.

[36] Peter Lucas Stöckli and Carmen Tanner. 2014. Are integrative or distributive outcomes more satisfactory? The effects of interest-based versus value-based issues on negotiator satisfaction. European Journal of Social Psychology 44, 3 (2014), 202-208.

[37] Hun-Tong Tan and Ken Trotman. 2007. Effects of auditors' concession timing on financial officers' negotiation judgments. Available at SSRN 960907 (2007).

[38] Leigh Thompson and George Loewenstein. 1992. Egocentric interpretations of fairness and interpersonal conflict. Organizational Behavior and Human Decision Processes 51, 2 (March 1992), 176-197.

[39] Kyle James Tusing and James Price Dillard. 2000. The sounds of dominance. Vocal precursors of perceived dominance during interpersonal influence. Human Communication Research 26, 1 (2000), 148-171.

[40] Rustam Vahidov, Gregory Kersten, and Raafat Saade. 2014. An experimental study of software agent negotiations with humans. Decision Support Systems 66 (2014), 135-145.

[41] Gerben A Van Kleef, Carsten KW De Dreu, and Antony SR Manstead. 2004. The interpersonal effects of anger and happiness in negotiations. Fournal of personality and social psychology 86, 1 (2004), 57.

[42] Tzu-Yang Wang, Ikkaku Kawaguchi, Hideaki Kuzuoka, and Mai Otsuki. 2018. Effect of Manipulated Amplitude and Frequency of Human Voice on Dominance and Persuasiveness in Audio Conferences. Proceedings of the ACM on HumanComputer Interaction 2, CSCW (2018), 177.

[43] Zhen Wang, John Lim, and Xiaojia Guo. 2010. Negotiator satisfaction in NSSfacilitated negotiation. Group Decision and Negotiation 19, 3 (2010), 279-300.

[44] Laurie R Weingart, Rebecca J Bennett, and Jeanne M Brett. 1993. The impact of consideration of issues and motivational orientation on group negotiation process and outcome. Fournal of Applied Psychology 78, 3 (1993), 504.

[45] Nancy A Welsh. 2003. Perceptions of fairness in negotiation. Marq. L. Rev. 87 (2003), 753.

[46] Scott Wiltermuth, Larissa Z Tiedens, and Margaret Neale. 2015. The benefits of dominance complementarity in negotiations. Negotiation and Conflict Management Research 8, 3 (2015), 194-209.

[47] Yang Yinping and Sharad Singhal. 2009. Designing an intelligent agent that negotiates tactfully with human counterparts: a conceptual analysis and modeling framework. In 2009 42nd Hawaii International Conference on System Sciences. IEEE, $1-10$. 